\title{
Optical Analysis of Cluster Mergers
}

\author{
Marisa Girardi \\ Dipartimento di Astronomia, Univ. di Trieste, via Tiepolo 11, I-34100 \\ Trieste, Italy
}

\begin{abstract}
An increasing amount of data has revealed that many clusters are very complex systems. Optical analyses show that several clusters contain subsystems of galaxies suggesting that they are still in the phase of relaxation, possibly after a phase of cluster merging. I briefly review the main results about substructure, and the connection between cluster dynamical status and galaxy properties. Useful comparisons with the results derived from X-ray data are also discussed.
\end{abstract}

\section{Cluster Substructure}

The first evidence that substructure might be very common in clusters comes from the galaxy density maps by Baier (1977 and following works) and the statistical analysis by Geller \& Beers (1982). More recently, both galaxy distribution and X-ray data are successfully combined to show evidence of cluster merging and constrain N-body simulations of cluster collisions (e.g., Zabludoff \& Zaritsky 1995 for the bimodal cluster ABCG 754). Also the velocity distribution of member galaxies gives evidence for cluster mergers (cf. the two-peaked clusters of Fadda et al. 1996) and can be combined with X-ray data.

The statistical methods used to quantify substructure can be grouped in three large families (see, e.g., Fitchett 1988 for a review; Pinkney et al. 1996; Girardi et al. 1997). The analysis of galaxy velocity distribution allows the detection of velocity substructure, but problems arise from the small number of available redshifts and the poor knowledge of the underlying velocity distribution. The analysis of spatial substructure takes advantage from the large number of data, but suffers from the contamination by fore/background galaxies. Finally, velocity-spatial substructure can be analyzed by combining redshifts and positions of cluster member galaxies. The higher the dimensionality of the test, the more sensitive it is to substructure (Pinkney et al. 1996). However, the sensitivity of tests depends on the line of sight relative to the merger axis, therefore calling for the need of using a battery of tests.

Present results, coming from the optical analysis of large cluster samples (out to $>100$ clusters), show that substructures are very frequent and concern $30-50 \%$ of clusters, most containing small size substructures, of size $\sim 0.2 h^{-1}$ Mpc and mass $10 \%$ of the parent cluster mass (e.g., Geller \& Beers 1982; Escalera et al. 1994; Girardi et al. 1997). Large scale substructures, like cluster bimodality, are less common and concern $\sim 10-20 \%$ of clusters (e.g., Girardi et al. 1997). The bimodality suggests a merging between two equal-mass clusters, 
while the situation is less clear for small size substructures, which can be the result of the accretion of small groups or the surviving dense core of merging clusters of similar masses (González-Casado, Mamon, \& Salvador-Solé 1994).

\section{Cluster Mergers and Large Structure}

Cluster mergers can be connected with the large scale structure of the Universe. There are examples of cluster complexes where the large number of clumps indicates that strong dynamical processes are active (e.g., the ABCG 3558 complex by Bardelli et al. 1998). In other cases, several galaxy systems are well aligned suggesting that they are falling onto each other along a preferential direction (e.g., the ABCG 85/87/89 complex by Durret et al. 1998), that is well in agreement with hierarchical cosmological scenarios, where matter, including galaxies, groups and gas, falls onto the cluster along a filament. In particular, the intersection of filaments are thought to be the natural place of rich cluster formation (cf. Arnaud et al. 2000 for ABCG 521).

\section{Cluster Dynamical Equilibrium and Global Properties}

Merging clusters are thought to depart strongly from dynamical equilibrium. Moreover, numerical simulations show that galaxy and gas components react with different time scales during a merger, e.g., two clusters can pass through one another without destroying individual optical components, while the gas is strongly affected (e.g., Roettiger, Loken, \& Burns 1997). Thus, merging clusters are expected to lie far from the typical relations between different global properties, in particular when comparing optical and $X$-ray properties (e.g., galaxy velocity dispersion, $\sigma_{v} ; \mathrm{X}$-ray luminosity, $L_{X}$, and temperature, $T_{X}$ ).

The presence of substructure is suggested to explain the departures from the $L_{X}-\sigma_{v}$ relation (e.g., for ABCG 1060, Fitchett 1988). Also high values of $\beta_{\text {spec }}=\sigma_{v}^{2} /\left(k T_{X} / \mu m_{p}\right)$, where $\mu$ and $m_{p}$ are the molecular weight and the proton mass, suggest the presence of substructure since $\beta_{\text {spec }} \sim 1$ if only gravitational processes are important (e.g., Edge \& Stewart 1991). Indeed, looking at all kinds of departures from the $\sigma_{v}-T_{X}$ relation can be very significant: e.g., $\beta_{\text {spec }} \sim 0.3$ for the bimodal cluster ABCG 754 (cf. Fadda et al. 1996).

Finally, some authors looked for discrepancies among mass estimates coming from different methods: virial analysis of optical data, $\mathrm{X}$-ray and gravitational lensing analyses. Recent results show that, avoiding bimodal clusters, there is a good agreement for large scale estimates, while some cases of strong discrepancy for central cluster regions still remain, possibly due to small substructures (e.g., Allen 1998; Girardi et al. 1998).

\section{Cluster Member Galaxies}

Merging clusters are strongly connected with properties of member galaxies. For instance, dominant galaxies in clusters $(\mathrm{cD}$ and $\mathrm{D}$ ) can be good tracers of cluster merging phenomena. In fact, although the formation theories of $\mathrm{cD} / \mathrm{D}$ require them to be located at the minimum of the cluster gravitational potential, $\mathrm{cD} / \mathrm{D}$ 
often show peculiar velocities, i.e., their velocity differs from the mean velocity of the rest of cluster galaxies. This peculiarity correlates with the presence of subclusters and disappears when $\mathrm{cD} / \mathrm{D}$ galaxies are assigned to their host subclumps (Bird 1994). This result and the evidence that $\mathrm{cD} / \mathrm{D}$ lie on local surface density peaks (Geller \& Beers 1982) suggest that dominant galaxies form in clumps before the virialization of parent clusters.

More in general, also bright galaxies can be good tracers for cluster mergers as shown by Biviano et al. (1996) in the analysis of the Coma cluster, where bright galaxies are located in two subclumps surrounding the two central dominant galaxies. A possible interpretation suggests that the dynamical evolution in groups goes on before their infall onto the clusters and leads to the formation of dense core-halos which will survive for a long time after the merging.

Moreover, there is some observational evidence that the environment of merging clusters is very effective on galaxy properties, abruptly truncating or enhancing the star formation rate. For instance, the fraction of blue galaxies correlates with cluster dynamical status (Wang \& Ulmer 1997), and the fraction of emission line galaxies is higher among galaxies belonging to subclusters (Biviano et al. 1997). In some cases, the $\mathrm{E}+\mathrm{A}$ galaxies, often interpreted as poststarbust galaxies, are spatially connected with the groups falling onto clusters (e.g., Caldwell et al. 1993 in the Coma cluster).

\section{References}

Allen, S. W. 1998, MNRAS, 296, 392

Arnaud, M., Maurogordato, S., Slezak, E., \& Rho, J. 2000, A\&A, 355, 461

Baier, F. W. 1977, Astr. Nachr., 298, 151

Bardelli, S., et al. 1998, MNRAS, 300, 589

Bird, C. M. 1994, ApJ, 422, 480

Biviano, A., et al. 1996, A\&A, 311, 95

Biviano, A., et al. 1997, A\&A, 321, 84

Caldwell, N., et al. 1993, AJ, 106, 473

Durret, F., et al. 1998, A\&A, 335, 41

Edge, A. C., \& Stewart, G. C. 1991, MNRAS, 252, 428

Escalera, E., et al. 1994, AJ, 423, 539

Fadda, D., et al. 1996, ApJ, 473, 670

Fitchett, M. J. 1988, ASP Conf. Ser., 5, 143

Geller, M. J., \& Beers, T. C. 1982, PASP, 94, 121

Girardi, M., et al. 1997, ApJ, 490, 56

Girardi, M., et al. 1998, ApJ, 505, 74

Gonzalez-Casado, G., Mamon, G., \& Salvador-Solé, E. 1994, ApJ, 433, L61

Pinkney, J., Roettiger, K., Burns, J. O., \& Bird, C. A. 1996, ApJS, 104, 1

Roettiger, K., Loken, C., \& Burns, J. O. 1997, ApJS, 109, 307

Wang, Q. D., \& Ulmer, M. P. 1997, MNRAS, 292, 920

Zabludoff, A., \& Zaritsky, D. 1995, ApJ, 447, L21 